Z Rheumatol 2009 $68: 195-195$ DOI 10.1007/s00393-008-0389-Z

Online publiziert: 29. April 2009

(c) Springer Medizin Verlag 2009

\author{
A. Radbruch ${ }^{1}$ G.-R. Burmester ${ }^{2}$ \\ ${ }^{1}$ Deutsches Rheumaforschungszentrum Berlin, Leibniz-Institut, \\ Charité-Universitätsmedizin Berlin \\ ${ }^{2}$ Medizinische Klinik mit Schwerpunkt Rheumatologie und Klinische Immunologie, \\ Charité-Universitätsmedizin Berlin
}

\title{
Stammzellen und Zelltherapie
}

Wie in den Beiträgen von Alexander und Schippling beschrieben, wissen wir heute aus den experimentiellen Therapien der Immunablation mit nachfolgende autologer Stammzelltransplantation bei systemischem Lupus erythematodes und bei multipler Sklerose, dass das Immunsystem sich aus Stammzellen regenerieren kann.

\section{$>$ Das Immunsystem kann sich aus Stammzellen regenerieren} phozyten durch Rituximab, aber auch die Immunablation durch Anti-Thymozytenglobulin. Wir beginnen zu verstehen, dass der Körper, insbesondere das Immunsystem, ein Gedächtnis für die rheumatische Entzündung entwickelt. Die Zellen dieses Gedächtnisses lassen sich offenbar durch die körpereigenen Regulationsmechanismen nicht mehr kontrollieren. Sie sind auch resistent gegen konventionelle Immunsuppression. Sie sind es offenbar, die nach dem Absetzen einer Biologika-Therapie die Entzündung erneut in Gang bringen können. Ihre Eliminierung ist Voraussetzung, um die rheumatische Entzündung dauerhaft $\mathrm{zu}$ beenden, den körpereigenen Regulationsmechanismen die Kontrolle zurückzugeben und so einen entscheidenden Schritt in Richtung „Heilung“ voranzukommen. Wir beginnen heute, das pathogene Gedächtnis des Immunsystems langsam zu verstehen - Voraussetzung für seine spezifische Eliminierung und damit für Therapien jenseits der kompletten Ablation des Immunsystems durch Anti-Thymozytenglobulin oder der Ablation der BLymphozyten durch Rituximab.
Es „verjüngt“ sich dabei quasi und wird wieder tolerant gegen den eigenen Körper. Die körpereigenen Regulationsmechanismen greifen also wieder. Wenn wir diese Regeneration verstehen, können wir auch gezielt neue Therapien entwickeln, die die körpereigene Immunregulation ausnutzen. Fritz Melchers beschreibt in seinem Beitrag die Grundlagen der Entwicklung von der Stammzelle zum Lymphozyten. Schon in der frühen Phase der Entwicklung werden normalerweise viele Zellen eliminiert, die den eigenen Körper erkennen. Gleichzeitig entstehen Zellen, die verhindern, dass autoreaktive Lymphozyten überhaupt aktiviert werden können. Solche regulierenden Zellen können Lymphozyten sein, wie in den Beiträgen von Alexander und Schippling diskutiert, oder aber Zellen des mesenchymalen Stromas, wie in dem Beitrag von Keyßer ausgeführt. Diese mesenchymalen Stromazellen sind auch noch aus einem anderen Grund von besonderem Interesse. Sie werden auch mesenchymale Stammzellen genannt, weil sie ein erstaunliches regeneratives Potenzial besitzen, wie in den Beiträgen von
Dehne und David beschrieben. Sie können sich zu einer Vielzahl von Zelltypen entwickeln, auch zu Chondrozyten und Osteoblasten. Mesenchymale Stammzellen sind also möglicherweise geeignet, um zerstörte Gewebe, z. B. Knochen und Knorpel, wieder aufzubauen, wenn denn die Entzündung einmal erfolgreich gestoppt worden ist. Zelltherapien bieten also Möglichkeiten, die weit über das hinausgehen, was heute möglich ist, einer Vision folgend, die erstmals und schon 1858 Rudolf Virchow in seiner „Cellularpathologie" formulierte.

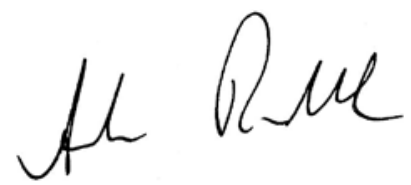

Andreas Radbruch

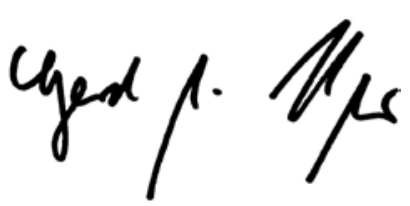

Gerd-Rüdiger Burmester

\section{Korrespondenzadresse \\ Prof. Dr. A. Radbruch}

Deutsches Rheumaforschungszentrum Berlin, Leibniz-Institut, Charité-Universitätsmedizin Berlin

Charitéplatz 1, 10117 Berlin

radbruch@drfz.de 